\title{
Clue to role of cannabinoid receptors in RA
}

New research pinpoints cannabinoid receptors as a potential new therapeutic target in rheumatoid arthritis (RA). The findings confirm that cannabinoid receptor 2 (CB2R) is expressed in synovial tissue and cultured fibroblastlike synoviocytes (FLS) from patients with RA, and that CB2R activation might have immunomodulatory and antiinflammatory effects in this setting.

"Historically, therapy with marijuana was used to ease the symptoms of a broad spectrum of diseases, including rheumatoid arthritis," explains author Sheng-Ming Dai from Second Military Medical University, China. "A major impediment to the widespread use of cannabinoids has been the psychotropic side effects," he adds. The discovery of cannabinoid receptors and selective agonists has garnered much interest for their potential clinical applications in inflammatory disease without these adverse effects.

CB2R is expressed by immune cells (with levels of expression varying among different cell types) and is thought to have a role in cannabinoid-mediated immune modulation. Reporting in Rheumatology (Oxford), Dai and colleagues investigated the expression and role of CB2R in RA.

\section{We ... provide evidence of} an available target for a CB2Rselective agonist in the treatment of RA... 77

The researchers confirmed that CB2R was expressed in synovial tissue samples and cultured FLS from patients with RA $(n=8)$ and in those with osteoarthritis $(n=4)$, although higher expression levels were observed in RA.

In cultured RA FLS, upon stimulation with proinflammatory mediators (TNF, IL-1 $\beta$ or lipopolysaccharide), levels of CB2R mRNA expression increased. Moreover, using a selective CB2R agonist (HU-308) in vitro, Dai et al. demonstrated anti-inflammatory aspects to CBR2 activation. Pre-treatment with HU-308 inhibited IL- $1 \beta$-induced proliferation of cultured RA FLS and production of proinflammatory cytokines (IL-6 specifically) and matrix metalloproteinases thought to be involved in cartilage erosion (MMP3 and MMP13). Interestingly, CB2R activation via HU-308 also suppressed IL-1 $\beta$-induced activation of MAPK signalling, most notably activation of p38 mitogen-activated protein kinase and ERK1/2.

"We provide evidence of an available target for a CB2R-selective agonist in the treatment of RA," explains Dai, who notes that their data also provide insights into the possible mechanisms of action. Further work is warranted to confirm these findings and how best to manipulate the endocannabinoid system in RA.

Katrina Ray

Original article Gui, H. et al. Expression of cannabinoid receptor 2 and its inhibitory effects on synovial fibroblasts in rheumatoid arthritis. Rheumatology (Oxford) doi:10.1093/rheumatology/ket447 